\title{
POLIMIOSITE E SEUS CUIDADOS: UM RELATO DE CASO
}

\author{
Kamilla Jessica de Oliveira ${ }^{1}$ \\ Francisca Kelly Silva de Andrade ${ }^{2}$ \\ Evelyn Heinzen ${ }^{3}$
}

RESUMO: A Polimiosite (PM) é uma doença idiopática sistêmica do sistema conjuntivo, que causa fraqueza muscular bilateral progressiva, através de processos inflamatórios não supurativos. É definida como uma patologia autoimune, crônica e sistêmica, podendo ou não estar associada a doenças autoimunes e infecções virais. Sua incidência é de o,5 a 8,4 casos por milhão de habitantes, tem prevalência duas vezes maior no sexo feminino. Seu principal aspecto é a morbidade e incapacidade funcional do indivíduo. O diagnóstico é feito através da alteração da enzima CPK, biopsia e ressonância nuclear magnética, quando não há alterações enzimáticas. O tratamento é realizado com o uso de corticoides e fisioterapia. Relataremos o caso de uma paciente portadora de Polimiosite para conhecer melhor a doença, pois os estudos quanto a esta patologia são escassos e quanto aos cuidados, não se têm publicações direcionadas.

Palavras-chave: Dermatomiosite. Polimiosite. Epidemiologia. Miopatia. Estudo de caso.

ABSTRACT: Polymyositis (PM) is an idiopathic disease systemic connective system, which causes muscle weakness bilaterally, through not supurativos inflammatory processes. Is defined as a chronic and systemic autoimmune pathology, which may or not be associated with autoimmune diseases and viral infections. Its incidence is 0.5 to 8.4 cases per million inhabitants, have twice the prevalence in females. Its main aspect is the morbidity and functional impairment of the individual. The diagnosis is made by amending the CPK enzyme, biopsy and MRI, when there is no enzyme changes. The treatment is performed with the use of steroids and physical therapy. We report the case of a patient with Polymyositis to learn more about the disease, because the studies about this pathology are scarce and as the care, have targeted publications.

Keywords: Dermatomyositis. Polymyositis. Epidemiology. Myositis. Case Studies.

\footnotetext{
I Pós- graduada em Urgência e emergência, Unidade de Terapia Intensiva e Nefrologia pela Faculdade Cerrado Brasília,DF. Docente no Centro Técnico de Educação Profissional de Águas Lindas,GO. Graduada em Enfermagem pelo Instituto de Educação Superior de Brasília. Enfermeira Voluntária PV no HOSPITAL REGIONAL DE CEILÂNDIA- PRONTO SOCORRO ADULTO. E-mail: kamilla_jessica@hotmail.com

${ }^{2}$ Pós- graduada em Cardiologia e hemodinâmica, Unidade de Terapia Intensiva e Nefrologia Clínica pela Faculdade CGESP. Graduada em Enfermagem pelo Instituto de Educação Superior de Brasília,.E-mail: kellysilvadeandrade@hotmail.com.

${ }^{3}$ Mestre em Gerontologia pela Universidade Católica de Brasília - UCB. Pós graduada em em Gestão de Serviço de Saúde e Auditoria, em Enfermagem em Cardiologia e em Doação de Órgãos e Transplante e Graduada em Enfermagem pela Universidade Católica de Brasília. Docente da Fundação de Ensino e Pesquisa em Ciências da Saúde do Distrito Federal e Enfermeira da Secretaria de Saúde do Distrito Federal SESDF.E-mail: evelyn.heinzen@fepecs.edu.br.
} 


\section{INTRODUÇÃO}

A Polimiosite (PM) e Dermatomiosite (DM) fazem parte de um grupo de miopatias inflamatórias idiopáticas, também chamadas de "Síndrome de Miosite" e das doenças sistêmicas adquiridas que acometem o sistema conjuntivo, causando processos inflamatórios musculares crônicos (ZEIGELBOIM et al; 20I0).

É definida como uma patologia autoimune, crônica e sistêmica, podendo ou não estar associada a doenças autoimunes e infecções virais, como lúpus, artrite reumatoide, doença de Crohn, vírus da imunodeficiência humana (HIV) e vírus linfotrópico de células T humanas (HTLV). Atinge o sistema muscular esquelético, causando fraqueza muscular bilateral progressiva, através de um processo inflamatório não supurativo, podendo apresentar acometimento articular cardíaco, pulmonar e trato gastrointestinal (MIRANDA et al, 2014).

Sua incidência é de 0,5 a 8,4 casos por milhão de habitantes, tem prevalência duas vezes maior no sexo feminino. Surge em sua maioria em indivíduos a partir dos 40 anos e possui pouca frequência na infância, tendo como principal aspecto a morbidade e incapacidade funcional do indivíduo (SHINJO et al, 2013, MISSUMI et al, 2014).

Tem como primeira sintomatologia a fraqueza muscular progressiva, sendo simétrica, bilateral e proximal, acometendo a musculatura da cintura escapular e pélvica. As queixas mais frequentes são dificuldade em realizar tarefas diárias, como subir no meio fio da calçada, levantar objetos e se pentear. Engasgos e disfagia podem estra presentes, relacionados ao acometimento da musculatura da faringe e esôfago (RACHID et al, 2005).

Em dois terços dos casos pode ocorrer comprometimento do flexor do pescoço, provocando dificuldade na sustentação do pescoço. Sintomas como, fadiga, hiporexia, perda ponderal, febre baixa, artralgia, ou artrite de pequenas e médias articulações podem estar presentes. Arritmias, taquicardia, defeitos de condução atrioventricular, e bloqueio de ramos podem estar presentes quando há o comprometimento cardíaco, insuficiência cardíaca congestiva, se apresenta com pouca frequência (FREIRE et al, 2010).

O diagnóstico é feito através da alteração da enzima CPK, que se altera em até 95\% em alguma fase da doença, biopsia muscular para a exclusão de outras doenças autoimunes e ressonância nuclear magnética, quando não há alterações enzimáticas. $\mathrm{O}$ 
tratamento é realizado com o uso de corticoides e fisioterapia na fase aguda ainda no leito, e com movimentação passiva nas articulações para evitar contraturas e encurtamento (DI GIACOMO et al, 2010).

Ao ter contato com um paciente portador de polimiosite, nos despertou o interesse neste estudo. Sabendo-se que a polimiosite provoca alto índice de morbidade e incapacidade funcional e os estudos são escassos.

Quanto aos cuidados, são raras as publicações direcionadas ao tema, é de extrema relevância, que hajam mais estudos e pesquisas sobre a temática, visando a compreensão da doença pelos profissionais de saúde e consequentemente o diagnóstico e tratamento precoce aos pacientes.

\section{OBJETIVOS}

\section{I Objetivo Geral}

Relatar um caso de polimiosite, abordando suas principais particularidades bem como sua progressão.

\subsection{Objetivos Específicos}

- Verificar características clínicas do portador da doença;

-Esclarecer como o diagnóstico foi realizado;

-Descrever o desfecho do tratamento utilizado;

-Estabelecer os cuidados de enfermagem, prestados a portadora da doença.

\section{REFERENCIAL TEÓRICO}

\section{I Polimiosite}

A Polimiosite (PM) é caracterizada como uma patologia autoimune sistêmica inflamatória idiopática, que provoca processos inflamatórios não supurativos e acomete o tecido muscular esquelético. Faz parte das miopatias inflamatórias idiopáticas, também denominadas como "síndrome de miosite". Está associada às enfermidades sistêmicas do tecido conjuntivo, tendo como característica principal a inflamação muscular crônica (ZEIGELBOIM et al., 20Io). 
Sua incidência varia geograficamente, de um caso por too mil habitantes, com predominância no sexo feminino. $\mathrm{Na}$ distribuição mundial ha um pico de prevalência, que se dá entre 50-60 anos de idade, com maior risco de desenvolvimento de miopatias inflamatórias idiopáticas entre indivíduos com descendência africana e hispânica, quando comparados a indivíduos de etnia branca, porém, na $(\mathrm{DM})$ e $(\mathrm{PM})$ não há predileção por etnia (SHINJO et al, 2013, MISSUMI et al., 2014).

\subsection{Sintomatologia}

Sua manifestação clínica se dá pela fraqueza muscular bilateral simétrica. $\mathrm{Na}$ literatura, estudos comprovam que pode ocorrer resposta anormal autoimune na musculatura esquelética. Como a sua fisiopatologia ainda é desconhecida, o mecanismo mais provável seria de causa muscular, por vírus ou de origem microvascular por auto antígenos (RACHID et al., 2005).

A doença age de forma gradual e progressiva, podendo ter meses ou anos de evolução. Há comprometimento da musculatura esquelética da parede posterior da faringe e do terço proximal do esôfago, dando origem à disfônia e a disfagia, ocasionando assim o refluxo de alimentos, para vias aéreas superiores pela fraqueza muscular cricofaríngea (ZEIGELBOIM et al., 2010).

Em dois terços dos casos há comprometimento do flexor do pescoço, causando dificuldade na sua sustentação, sobretudo, fraqueza muscular, que é seu sintoma principal (FREIRE et al., 20Io).

A musculatura da cintura escapular e pélvica também é comprometida, progredindo para a musculatura proximal dos membros. Os demais sintomas observados são: fadiga, astenia, mialgias, febre, perda de peso, artralgia e artrite de pequenas e médias articulações. Também há comprometimento cardíaco, estando presente em 30\% dos casos, causando taquicardia, arritmias, defeitos de condução atrioventricular e bloqueios de ramos (ZEIGELBOIM et al., 20Io).

Com pouca frequência, há insuficiência cardíaca congestiva, por miocardite ou por fibrose miocárdica. Manifestações pulmonares são as mais comuns da doença, com prevalência de $46 \%$ dos casos, as mais observadas são pneumonia aspirativa, 
hipoventilação por fraqueza muscular, infecções e doença intersticial (FREIRE et al, 2oı́; ZEIGELBOIM et al., 2010).

\subsubsection{Diagnóstico}

O diagnóstico das miopatias inflamatórias é realizado através do quadro clínico do paciente, pelo surgimento da fraqueza muscular e exames laboratoriais como: creatinofosfoquinase (CPK), aldolase, desidrogenase lática (LDH), alaninoamiotransferase (ALT) e aspartatoaminotransferase (AST), exame eletroneuromiográfico, ressonância magnética, biópsia muscular e como se dá à resposta ao tratamento da doença (SOUZA et al., 20II; DI GIACOMO et al., 20ıo).

A RM (Ressonância magnética) é dos métodos de imagem disponíveis atualmente, o mais sensível para deteç̧ão de alterações. Através da RM é permitido avaliar todas as estruturas, incluindo partes moles, ossos, cartilagens e detectar erosões até três anos antes. Através da RM é possível avaliar o local das alterações observadas, que possam ter implicação prognóstica (MOTA et al., 2012).

A ENMG (eletroneuromiografia) é um método de acompanhamento das manifestações bioelétricas, que ocorrem nas membranas das células e das fibras musculares esqueléticas (HELFENSTEIN JR, 2013).

\subsubsection{Tratamento}

A falta de diagnóstico direcional, dificulta a condução de um tratamento terapêutico padronizado, fazendo-se necessário uma abordagem individualizada quanto à gravidade de cada paciente, diante do seu quadro clinico. $O$ tratamento não farmacológico é feito através dos programas de reabilitação, desde o início da doença (DI GIACOMO et al., 2010; BRASIL, 2010).

O tratamento farmacológico é iniciado com glicocorticoide sistêmico, devendo ser informado ao paciente quanto à duração que poderá ser superior a 12 meses. Seu uso poderá induzir remissão da doença em $40 \%$ - 60\% dos pacientes, sendo que mais de $80 \%$ apresentam algum grau de melhora clínica e laboratorial (BRASIL, 2010). 
Ainda segundo o Protocolo Clínico e Diretrizes Terapêuticas, publicado na Portaria SAS/MS no 206, de 23 de abril de 2010, a duração do tratamento poderá ser realizada conforme a evolução clínica da patologia, ou alterações detectadas diante da monitorização da toxicidade dos medicamentos.

\subsection{Cuidados de enfermagem}

\subsubsection{Evolução do cuidado da enfermagem}

Desde os primórdios da humanidade, o cuidado faz parte da vida do ser humano, como resposta de atendimento às suas necessidades. Para o cuidado ser realizado, deverá o enfermeiro, como um membro participante da equipe multidisciplinar, utilizar um conjunto de conhecimentos que o possibilitem a busca de resoluções para a manutenção e recuperação da saúde (BALDUINO et al., 2009).

Os pioneiros da enfermagem eram pessoas religiosas que forneciam assistência por caridade para pessoas doentes e carentes, nessa época, o cuidado prestado era de caráter religioso e sem base científica. A Sistematização da Assistência de Enfermagem (SAE) surgiu para que sua aplicação seja realizada como forma de um instrumento metodológico para possibilitar a equipe de enfermagem aplicar o processo de enfermagem e prognosticar, como o paciente responde aos problemas de saúde e possibilitar a determinação de como essas respostas exigirão uma intervenção (MEDEIROS et al., 2012).

A intervenção de enfermagem é definida como: cuidados baseados através do conhecimento clínico e científico, exercida pela equipe de enfermagem, como resultado obtido para o paciente, devendo ocorrer a partir da criação de um diagnóstico de enfermagem para classificar os cuidados prestados como uma ação padrão para proporcionar a sistematização e da assistência prestada pela enfermagem. Ao planejar e programar o cuidado de enfermagem individualizado, no auxilio efetivo para melhoria da saúde, as intervenções devem ser realizadas em diferentes situações para cuidado, com métodos e instrumentos variados, aplicados aos pacientes (SCAIN et al., 2013).

Os cuidados de enfermagem devem contribuir para o bem-estar integral dos indivíduos. No entanto, para a prestação deste cuidado, o enfermeiro também necessita de conhecimentos e fundamentos dos conceitos que existem nas relações biológicas, psicossociais, e suas implicações psicológicas e afetivas, dos envolvidos no cuidado de enfermagem (SEIMA et al., 2oIr; SILVA, TEIXEIRA, 20II). 
O cuidado de enfermagem tem adquirido novos significados, influenciado por novas abordagens, com isso, o cuidado passa a ser entendido como fenômeno motivado pelo desenvolvimento a partir de redes de saberes inter e transdisciplinar. O cuidado como perspectiva da complexidade permite a compreensão de que, para cuidar de forma integral é indispensável à compreensão de ações e saberes dos profissionais da saúde, como forma de valorizar cada ser humano envolvido. Uma das atividades fundamentais da enfermagem na prática da saúde é o cuidado que deve ser compreendido como, sistema complexo e integrador (PIEXAK et al., 2013).

\subsection{2 $\mathrm{O}$ cuidado de enfermagem na Polimiosite}

Quanto a Polimiosite e Dermatomiosite os cuidados ainda são poucos descritos, porém enfatiza-se que segundo o Protocolo Clínico e Diretrizes Terapêuticas, publicado na Portaria SAS/MS no 206, de 23 de abril de 2010, existem quatro condutas principais a serem adotadas, a primeiras delas é a orientação quanto as medidas para evitar aspiração em pacientes com disfunção esofágica.

A broncoaspiração de secreção e resíduos alimentares pode ocasionar várias

patologias, entre elas as infecções pulmonares, a pneumonite química ou síndrome da angústia respiratória, com morbidade e mortalidade significativas (Beck-Schimmer e Bonvini, 20II). Estudos recomendam que mudanças no cardápio alimentar com ajustes de acordo com o estado crítico do paciente, estão entre os parâmetros de prevenção, levantar a cama em um ângulo de $45^{\circ}$ é um dos itens principais dos cuidados aos pacientes de risco (Saez de la Fuente et al., 2016); (ALMEIDA et al., 2016).

Posicionar o paciente em decúbito lateral esquerdo elevado, não deitar logo após as refeições e não consumir alimentos ácidos ou gordurosos podem ter efeitos benéficos durante o tratamento, e prevenir diversas complicações como pneumonias, sinusites e as internações constantes, diminuindo a angústia dos familiares (CORDEIRO et al., 2014).

A segunda conduta a ser adotada pelo protocolo é quanto à foto proteção. A Organização Mundial de Saúde prevê, como medidas de proteção, restringir a exposição solar em horários aproximado as I2:00 h, optar lugares com sombras, utilizar vestimentas de proteção e óculos solar, utilizar protetor solar todos os dias e reaplicá-lo de duas em duas 
horas, ou depois do trabalho, nado, jogos ou ao se exercitar ao ar livre (PURIM,WROBLEVSK, 2014).

O uso do protetor solar é uma estratégia eficaz na redução de agravos à saúde ocasionados pela radiação ultravioleta. Associar o uso de foto proteção química, com uso de barreira física, como vestimentas de manga comprida, bonés, óculos escuros e o cuidado com relação ao horário de exposição ao sol, não se expondo no período entre to e 15 horas, mostram-se mais eficientes para diminuir a ocorrência de danos à pele (LIMA et al., 2010).

Quanto à prevenção da osteoporose, a atividade física é o meio mais econômico, a prática desses exercícios físicos associados ao uso de peso e velocidade mostram-se mais eficientes para o ganho de massa óssea. A reposição hormonal e estrogênica também funciona como prevenção para osteoporose (SOUZA et al., 2010).

Por último, a prevenção de infecções oportunistas, descrita pelo protocolo e realizada através da reposição de vitamina $D$, pois de maneira geral, seu efeito no sistema imunológico se manifesta através do aumento da imunidade inata, agregada a uma regulação da imunidade adquirida. Os estudos realizados ainda são escassos e não controlados, contudo também parecem mostrar que a suplementação da vitamina $\mathrm{D}$ tem efeitos benéficos na prevenção do desenvolvimento de doenças autoimunes, além disso, apontam uma redução no agravo da doença preexistente (SOUZA et al., 2010).

Estudos evidenciam que a deficiência de vitamina $\mathrm{D}$ pode ter um papel de grande relevância na regulação do sistema imunológico e, supostamente, na prevenção das doenças imunomediadas. Entretanto, ainda são recomendados mais estudos para determinar os riscos e benefícios da reposição de vitamina D (MARQUES et al., 2oro).

As pessoas portadoras de doenças reumatológicas crônicas, apresentam um risco de infecção duas vezes maior do que a população geral. O risco se dá, tanto pela imunossupressão decorrente da doença, quanto pela terapia medicamentosa empregada. A vacinação se mostra eficaz na redução da morbimortalidade em pacientes com doenças reumatológicas, em tratamento imunossupressor. As vacinas feitas com vírus e bactérias mortos ou de proteínas ou açúcares desses patógenos mostraram-se seguras nesse grupo de pacientes (MOTA et al., 2009). 


\section{METOdOlOGiA}

\section{I Tipo de estudo}

Trata-se de um estudo descritivo, com uma abordagem qualitativa, do tipo estudo de caso, que é uma modalidade de estudo que permite uma investigação significativa da polimiosite. Este tipo de estudo ainda contribui, de maneira inigualável, para a compreensão tanto para o portador da doença quanto para profissionais da área da saúde.

\subsubsection{Amostra}

O estudo foi realizado com uma paciente portadora de Polimiosite que aceitou participar da pesquisa mediante a assinatura do Termo de Consentimento Livre e Esclarecido.

\subsubsection{Instrumento de coleta de dados}

A coleta de dados foi realizada através de um questionário. Na pesquisa ainda serão utilizadas algumas informações como; resultados de exames, o depoimento da paciente e fotos.

\subsubsection{Procedimento de coleta de dados}

Os dados foram coletados através de uma entrevista semiestruturada guiada por um roteiro padronizado contendo a descrição de como foi o desenvolvimento da doença, evidenciando suas principais características e progressão.

\subsubsection{Análise de dados}


Foram relatadas nesta pesquisa as características clínicas da Polimiosite como; quais sintomas levaram a paciente a procurar assistência médica, fazer a descrição de como foi realizado o diagnóstico da doença, qual foi o tratamento utilizado para a doença e quais cuidados de enfermagem devem ser realizados para essa paciente.

\section{4.r.6 Aspectos éticos}

O trabalho foi aprovado pelo Comitê de Ética em Pesquisa do Centro Universitário de Brasília (CEP-UniCEUB), sob o CAAE:67265317.6.oooo.oo23. O desenvolvimento da pesquisa está de acordo com o protocolo avaliado e aprovado, e em atenção às diretrizes éticas nacionais quanto aos incisos XI.I e XI.2 da resolução $\mathrm{n}{ }^{-} 466 / \mathrm{I2} / \mathrm{CNS} / \mathrm{MS}$ concernentes às responsabilidades do pesquisador no desenvolvimento do projeto.

\section{RESULTADOS}

A seguir apresentam-se os resultados de acordo com os objetivos propostos no presente estudo, estes emergiram do relatório desenvolvido no estudo de caso, bem como da observação direta, das entrevistas e do questionário.

\section{I Coleta de informações}

As informações foram coletadas no dia 22 de agosto de 2017 , pelo período da tarde, com duração de 2 horas. As informações foram obtidas por meio da aplicação de um questionário e análise de exames. Foi utilizado como instrumento para coleta de dados um questionário, observação direta e exames realizados pela paciente.

\subsection{Relato de caso}


Paciente do sexo feminino, 33 anos de idade, parda, empregada doméstica, natural de Brasília DF, com diagnóstico de Polimiosite há dois anos, em uso de Rituximab e Prednisona $80 \mathrm{mg}$, com quadro clínico e laboratorial compensado há oito meses.

Relatou que, os sintomas iniciais da doença foram fraqueza muscular proximal em cinturas escapular e pélvica (dificuldade ao subir escadas, se pentear, se abaixar e levantar), incapacidade de deglutição, perca de peso, disfagia, dispnéia mialgia, astenia, náuseas, vômitos e picos febris. Alega comorbidades como, febre reumática desde os nove anos com sequela cardíaca, e epilepsia desde a infância.

Foi diagnosticada um ano e oito meses após procura médica através dos exames, fator antinuclear (FAN), CPK e ressonância nuclear magnética, desde então, apresentou um quadro de insuficiência cardíaca congestiva, com boa resposta ao uso de furosemida e atenolol $25 \mathrm{mg}$ uma vez ao dia.

As principais limitações da doença se dão através da dificuldade em realizar tarefas mínimas como: locomoção, higienização, alimentação, escrever, abaixar, levantar, subir degraus, dificuldade de concentração e até mesmo para falar. As orientações recebidas pela equipe de enfermagem se deram, pelo risco de aspiração e ao uso de proteção solar. A pacien te relata que não recebeu nenhuma orientação quanto ao risco de queda.

A paciente relatou receio quanto ao futuro por temer a desestabilização e agravamento do quadro da doença. O recado que daria aos portadores dessa doença, seria realizar o tratamento corretamente, para uma melhor qualidade de vida.

A paciente relatou temer o agravamento da doença, por conhecer a evolução clínica da doença e o medo de desestabilização do quadro.

\footnotetext{
[...] Eu acho que quem tem polimiosite, tem que fazer o tratamento certo, seguir tudo direitinho, pra doença não piorar, para viver uma vida melhor (J.C.S., 2017)
}

\section{DISCUSSÃO}

A literatura apresenta poucas publicações sobre a PM. Na pesquisa bibliográfica realizada foram encontrados poucos artigos sobre a temática, quanto aos cuidados de 
enfermagem, não se tem publicações direcionadas ao tema, o que nos levou a estudar o presente caso.

Os achados clínicos e laboratoriais descritos para essa paciente, de 33 anos de idade, incluem o quadro de fraqueza muscular proximal, característico da PM. Com o aparecimento da fraqueza muscular proximal dos membros superiores e inferiores, associado à elevação da $\mathrm{CPK}$ e histológicos marcadores típicos de miopatia inflamatória, firmou-se o diagnóstico de PM.

Ao observarmos, foi possível perceber, através dos sintomas relatados pela paciente e da breve literatura descrita, que a teoria se estende a prática, ou seja, os sintomas manifestados na paciente foram os mesmos descritos nas literaturas, como: fraqueza muscular, incapacidade de deglutição, perca de peso, disfagia, dispnéia, mialgia, astenia, náuseas, vômitos e picos febris.

As orientações prestadas pela equipe de enfermagem, contribuem de forma complementar, na prevenção dos agravos e, na manutenção da qualidade de vida dos pacientes portadores da PM. A terapia medicamentosa e as orientações de enfermagem,

trouxeram uma melhora ao quadro clínico e laboratorial da paciente, mantendo uma evolução clínica favorável.

Assim sendo, esse relato representa a descrição de um caso de PM, abordando a importância dos cuidados prestados pela equipe de enfermagem e como curiosidade, o fato de a paciente ter apenas 31 anos de idade, quando se descobriu a doença, estando fora da incidência descrita na literatura.

\section{REFERÊNCIAS}

ALMEIDA, Ana Elizângela do Monte; ALCÂNTARA. J. bras. econ. saúde (Impr.); 8(3): 216-220, I0/o2/2017. Disponível ehttp://docs.bvsalud.org/biblioref/2017/o2/831902/jbes83ao6-prevalencia-de-risco-moderado-e-alto-de-aspiracao.pdf. Acessos em 17 mar. 2017

BRASIL. Portaria SAS/MS no 1.692 , de 22 de novembro de 2016. Protocolo Clínico e Diretrizes Terapêuticas Dermatomiosite e Polimiosite. Diário Oficial da União no ${ }^{226}$, Seção I, pág. 39, 25 novembro 2016. 
CORDEIRO, Jacqueline Andréia Bernardes Leão et al . Dificuldades enfrentadas pelos pais de crianças com doença do refluxo gastroesofágico. Acta paul. enferm., São Paulo, v. 27, n. 3, p. 255-259, jun. 2014 . Disponível em $<$ http://www.scielo.br/ \&pid=Soro321002014000300255\&lng=pt\&nrm=iso $>$. acesso em: 05 abr. 2017.

Di Giácomo, Carla Gaspar; Bueno, Adrian Nogueira; Vaz, João Luiz Pereira; Salgado, Maria Cecília da Fonseca.Rev. Soc. Bras. Clín. Méd; 8(5)set.-out. 2010.

DUARTE, Sabrina da Costa Machado et al . O cuidado de enfermagem no pós-operatório de cirurgia cardíaca: um estudo de caso. Esc. Anna Nery, Rio de Janeiro , v. i6, n. 4, p. 657-665, dez. 2012 . Disponível em: <http://www.scielo.br/ \&pid=Si4I481452012000400003\&lng=pt\&nrm=iso $>$. acessos em: is mar. 2017.

FREIRE, Renato Oliveira; MACIEIRA, José Caetano e BRITO, Hugo Leite de Farias. Polimiosite associada à síndrome nefrótica. Rev. Bras. Reumatol. [online]. 2010, vol.50, n.4, pp.462-463. ISSN 0482-5004.

HELFENSTEIN JR, Milton et al . Ultrassonografia no diagnóstico da tendinite e eletroneuromiografia no diagnóstico da neuropatia periférica e da radiculopatia do membro superior: visão do reumatologista. Rev. Bras. Reumatol., São Paulo , v. 53, n. 3, p. 282-287, jun. 2013 . Disponível em: 〈http://www.scielo.br/ =So48200oo6\&lng=pt\&nrm=iso $>$. acesso em: io mar. 2017.

LIMA, Angélica Gomes et al. Fotoexposição solar e fotoproteção de agentes de saúde em município de Minas Gerais. Revista Eletrônica de Enfermagem, Goiânia, v. I2, n. 3, p. 47882, set. 2010. ISSN I518-1944. Disponível em: 〈https://www.revistas.ufg.br/fen/article/view/6i56〉. Acesso em: o5 abr. 2017. doi:http://dx.doi.org/10.5216/ree.vi2i3.6156.

MARQUES, Cláudia Diniz Lopes et al . A importância dos níveis de vitamina D nas doenças autoimunes. Rev. Bras. Reumatol., São Paulo, v. 50, n. I, p. 67-8o, fev. 2010 . Disponível em $\quad\langle$ http://www.scielo.br/scielo.php?script=sci_arttext\&pid=So48250042010000100007\&lng=pt\&nrm=iso $>$. acesso em: 19 mar. 2017.

MIRANDA, Sofia Silveira de Castro; ALVARENGA, Daniel; RODRIGUES, João Carlos e SHINJO, Samuel Katsuyuki. Aspectos distintos de ressonância magnética de músculos entre dermatomiosite e polimiosite. Rev. Bras. Reumatol. [online]. 2014, vol.54, n.4, pp.295-300. ISSN 0482-5004. 
MISSUMI, Larissa Sayuri; SOUZA, Fernando Henrique Carlos de; ANDRADE, Joelma Queiroz e SHINJO, Samuel Katsuyuki. Desfechos da gestação em pacientes com dermatomiosite e polimiosite. Rev. Bras. Reumatol. [online]. 2015, vol.55, n.2, pp.95-102. ISSN 0482-5004.

MOTA, Licia Maria Henrique da et al . Diagnóstico por imagem da artrite reumatoide inicial. Rev. Bras. Reumatol., São Paulo, v. 52, n. 5, p. 761-766, out. 2012. Disponível em $<$ http://www.scielo.br/scielo.php?script=sci_arttext\&pid=So482$50042012000500010 \& \operatorname{lng}=\mathrm{pt} \& \mathrm{nrm}=$ iso $>$. acesso em: Io mar. 2017.

MOTA, Licia Maria Henrique da et al . Vacinação contra febre amarela em pacientes com diagnósticos de doenças reumáticas, em uso de imunossupressores. Rev. Soc. Bras. Med. Trop., Uberaba, v. 42, n. I, p. 23-27, fev. 2009. Disponível: 〈http://www.scielo.br/.ph >. acessos em: 17 mar. 2017 .

PIEXAK, Diéssica Roggia; BACKES, Dirce Stein; SANTOS, Silvana Sidney Costa. Cuidado de enfermagem para enfermeiros docentes na perspectiva da complexidade. Rev. Gaúcha Enferm., Porto Alegre, v. 34, n. 2, p. 46-53, jun. 2013 . Disponível em $\langle$ http://www.scielo.br/scielo.php?script=sci_arttext\&pid=SI983-I4472013000200006\& >. acessos em: Io mar. 2017.

PURIM, Kátia Sheylla Malta; WROBLEVSKI, Fernanda Cristina. Exposição e proteção solar dos estudantes de medicina de Curitiba (PR). Rev. bras. educ. med., Rio de Janeiro , v. 38, n. 4, p. 477-485, dez. 2014 . Disponível em <http://www.scielo.br/scielo. \&pid=So100-55022014000400009\&lng=pt\&nrm=iso $>$. acesso em 17 mar. 2017.

RACHID, Acir et al . Um caso atípico de polimiosite com acometimento da língua. Rev. Bras. Reumatol., São Paulo , v. 45, n. 4, p. 270-272, Aug. 2005 . Available from <http://www.scielo.br/scielo. \&pid=So482-50042005000400015\& >.accesson 03 Sept. 2016.

SCAIN, Suzana Fiore et al . Acurácia das intervenções de enfermagem para pacientes com diabetes mellitus tipo 2 em consulta ambulatorial. Rev. Gaúcha Enferm., Porto Alegre , v. 34, n. 2, p. 14-20, jun. 2013 . Disponível em <http://www.scielo.br/scielo.p \&pid=Sig83I4472013000200002\&lng=pt\&nrm=iso $>$ acessos em is mar. 2017.

SEIMA, Márcia Daniele et al. A produção científica da enfermagem e a utilização da teoria de Madeleine Leininger: revisão integrativa 1985 - 201ı. Esc. Anna Nery, Rio de Janeiro , v. 15, n. 4, p. $851-857$, dez. 20II . Disponível em 
$\langle$ http://www.scielo.br/scielo.php?script=sci_arttext\&pid=SI4I4-8I452011000400027iso $>$. acessos em is mar. 2017.

SHINJO, Samuel Katsuyuki e SOUZA, Fernando Henrique Carlos de. Atualização na terapêutica da calcinose em dermatomiosite. Rev. Bras. Reumatol. [online]. 2013, vol.53, n.2, pp.2II-214. ISSN 0482-5004.

SILVA, Carlos Magno Carvalho da; TEIXEIRA, Enéas Rangel. Exame físico e sua integralização ao processo de enfermagem na perspectiva da complexidade. Esc. Anna Nery, Rio de Janeiro, v. I5, n. 4, p. 723-729, dez. 20II . Disponível em 〈http://www.scielo.br/scielo.php?sc -81452011000400oio\&lng=pt\&nrm=iso . acessos em is mar. 2017.

SOUZA, Fernando Henrique Carlos de; LEVY-NETO, Maurício e SHINJO, Samuel Katsuyuki. Prevalência de manifestações clínico-laboratoriais e comorbidades na polimiosite segundo o gênero. Rev. Bras. Reumatol. [online]. 20II, vol.51, n.5, pp.428-483. ISSN 0482-5004

SOUZA, Márcio Passini Gonçalves de. Diagnóstico e tratamento da osteoporose. Rev. bras. ortop., São Paulo, v. 45, n. 3, p. 220-229, June 2010 . Available from 〈http://www.scielo.br/scielo.php? -36162010000300002\&lng=en\&nrm=iso〉. access on i9 Mar. 2017.

ZEIGELBOIM, Bianca Simone et al. Achados otoneurológicos em um caso de miopatia inflamatória. Rev. CEFAC [online]. 2012, vol.I4, n.I, pp.I7I-175. Epub II-Jun-2010. ISSN 1982-0216 